\title{
Ist Coaching auch für erfahrene Führungskräfte nützlich?
}

\section{Astrid Schreyögg}

Die meisten Menschen vermuten, dass Coaching in erster Linie von jungen, unerfahrenen Führungskräften für ihre ersten Schritte in Organisationen genutzt wird. Und tatsächlich finden wir heute vermehrt Publikationen, die sich mit dem Coaching von Newcomern in der Führung oder mit neu ernannten Führungskräften befassen (vgl. Fischer 2015; Goldfuß 2006; Kellner 2001; Schreyögg 2010 u. a.). Im Verlauf der letzten Jahre, während derer sich Coaching als Beratungsform immer stärker etabliert hat, erhielten aber zunehmend auch ältere oder schon etablierte Führungskräfte die Möglichkeit, einen Coach zu engagieren. Vor allem Systeme, die, wie etwa VW, schon über jahre- oder gar jahrzehntelange Erfahrungen mit dem Format Coaching verfügen, bieten ihren langjährigen Mitarbeitern von sich aus die Möglichkeit, sich coachen zu lassen.

So bot z. B. die Firma Audi deutschlandweit im Rahmen einer Exzellenzkampagne allen Geschäftsführern ihrer Autohäuser an, sich für ca. sieben Sitzungen coachen zu lassen. Bei diesen Autohäusern handelte es sich meistens um Unternehmen, die von Eigentümern geführt wurden, also um Familienunternehmen. Und diese verfügten zwar über ein enormes Kompetenzpotenzial operativer Art, verharrten aber als Manager zum Teil auf Routinen, die sich nicht immer als funktional erwiesen. Durch Coaching gelang es dann häufig, wenig sinnvolle Muster zu lockern oder gar aufzulösen. Als besonders sinnvoll erwies sich das Coaching bei Generationen übergreifenden Themen wie z. B. solchen: „Mein Opa sagt immer, ich soll alles so machen wie er". Dann ging es darum, dem Opa zu helfen, dass er lernt, seiner Enkelin zu vertrauen. Denn als junge Frau wollte sie auf dem Hintergrund neuartiger Ausbildungen selbstverständlich auch andere Wege beschreiten als der Großvater.

Coaching für ältere Mitarbeiter finden wir heute allerdings auch in Behörden wie etwa in der Bundesagentur für Arbeit oder in Ministerien. Auch in diesen Systemen hat

Dr. A. Schreyögg ( $\bowtie)$

Breisgauer Str. 29,

14129 Berlin, Deutschland

E-Mail: info@schreyoegg.de 
sich Coaching seit etlichen Jahren vor allem als organisationsinterne Maßnahme gut etabliert. Hier besteht dann auch eine geringe Hürde für ältere Mitarbeiter, Coaching in Anspruch zu nehmen, denn der „Coach sitzt ja gleich nebenan“. Dies geschieht oft im Anschluss an Fortbildungsseminare, während derer irgendein Veränderungsbedarf zu Tage trat. Und dieser kann dann im Coaching weitergehend bearbeitet werden.

Im ersten Beitrag umreißt Markus Borck, Wirtschaftspsychologe und Coach in einer Bundesbehörde, ein breites thematisches Spektrum, wie ältere, erfahrene Führungskräfte für sich und für die Organisation, in der sie tätig sind, durch Coaching profitieren können. Er plädiert dafür, dass Organisationen älteren Mitarbeitern Coaching aktiv offerieren, damit sie sich aus festgefahrenen Routinen lösen, wieder neu motiviert werden, die eigene Führungsphilosophie reflektieren usw. Diese Form von "Angebots-Coaching“" wird sich in den nächsten Jahren vermutlich immer stärker durchsetzen. Auf diese Weise lässt sich übrigens auch die Überalterung der Mitarbeiterschaft vieler Unternehmen günstig beeinflussen.

Im nachfolgenden Aufsatz zeigt Uta Lärm, Organisationspsychologin, dass die Personalverantwortung im Milieu der öffentlichen Verwaltung enorm zugenommen hat. Auch hier wurde wie in vielen Organisationen der Wirtschaft in großem Umfang das sogenannte Lean Management durchgesetzt. Dadurch gilt es heute aber anders zu führen als noch vor 20 Jahren. Das heißt, traditionelle Formen von Anweisung und Kontrolle sind jetzt praktisch außer Kraft gesetzt. Einer nun größeren Zahl von unterstellten Mitarbeitern als früher muss die Führungskraft notwendigerweise Vertrauen entgegenbringen, dass sie ihre Arbeit „nach bestem Wissen und Gewissen“ ausführen. Das aber führt zu erheblichen Irritationen, die bis in die Privatwelt hinein reichen können. Und von so einem Beispiel berichtet dann die Autorin ausführlicher.

Wie Jutta Müller, Elmar Schwedhelm und Dirk Bayas-Linke darstellen, sind Führungskräfte im Laufe ihrer beruflichen Entwicklung immer wieder mit Fragen konfrontiert, wie sie ihre Karriere gezielt vorwärts bringen können. Und dafür bedienen sie sich vielfach eines Karrierecoachings. Gerade erfahrene Führungskräfte tun dies aber gern „unter ihresgleichen“, d. h. im Rahmen kollegialer Beratung. Hier ergibt sich dann vielfach ein hohes $\mathrm{Ma}$ an Offenheit und methodischer Experimentierbereitschaft. Die Autoren präsentieren hier ein Workshop-Format mit narrativen Interviews, bei dem die erzählte Lebensgeschichte und damit biographische Strukturen in die kollegiale Beratung einfließen.

Eva-Maria Lewkowicz und Beate West-Leuer berichten ebenfalls vom Coaching mit erfahrenen Führungskräften. In ihrem Beitrag geht es um Führungskräfte in multinationalen, börsennotierten Unternehmen. Diese haben heute zunehmend in hochkomplexen, oft schwer durchschaubaren Strukturen und Netzwerken zu arbeiten. Da ergeben sich vielfältige Konflikte um Weisungsbefugnisse innerhalb von Matrixoder Projektorganisationen. Die Autorinnen zeigen an einem Praxisbeispiel, welche Besonderheiten dann im Business-Coaching zu beachten sind.

Die folgenden Beiträge sind anderen Themenbereichen gewidmet. Im Beitrag von Christiane Wenhart geht es um ein Marketingthema, wie nämlich durch Coaching Markenberatung verbessert werden kann. Die Autorin berichtet von einer Internetrecherche, die 40 Berater von Agenturen und Beratungen mit dem Fokus auf Marke, Marketing, Kommunikation, Design usw. umfasste. Es zeigte sich, dass zumal in 
größeren Agenturen selten ein spezialisiertes Coaching genutzt wird, das auf strategische Verbesserungen einer Marke abzielt.

Beata Williams, Achim Schneider und Petra A. Arndt präsentieren einen Praxisbericht, in dem sie nach den Effekten fragen, die Wissenschaftler/innen bei der Prozessunterstützung in der Veränderung von Bildungskontexten erzeugen. Dabei handelt es sich um die Prozessbegleitung des baden-württembergischen Landesmodells „Bildungshaus 3-10“.

Im Diskurs rollt Karl Kreuser, nach seiner vierjährigen Forschungstätigkeit Fragen auf, inwieweit sich Coaching und Mediation ähneln oder unterscheiden. Der Autor meint, dass sie sich vielfach ausgesprochen gut ergänzen.

\section{Literatur}

Fischer, P. (2015). Neu auf dem Chefsessel. München: Redline.

Goldfuß, J. W. (2006). Endlich Chef-was nun? Frankfurt a. M.: Campus.

Kellner, H. (2001). Karrieresprung durch Selbstcoaching. Frankfurt a. M.: Campus.

Schreyögg, A. (2010). Coaching für die neu ernannte Führungskraft. Wiesbaden: Springer VS. 